\title{
Self-Consistent Pedestal Prediction for JET-ILW in Preparation of the DT Campaign
}

\author{
S. Saarelma ${ }^{1}$, L. Frassinetti ${ }^{2}$, P. Bilkova ${ }^{3}$, C.D. Challis ${ }^{1}$, A. Chankin $^{4}$, R. Fridström ${ }^{2}$, L. \\ Garzotti $^{1}$, L. Horvath ${ }^{1,5}$, C.F. Maggi ${ }^{1}$ and JET Contributors* \\ ${ }^{1}$ Culham Centre for Fusion Energy, Culham Science Centre, Abingdon, OX14 3DB, UK \\ ${ }^{2}$ Division of Fusion Plasma Physics, KTH Royal Institute of Technology, Stockholm SE \\ ${ }^{3}$ Institute of Plasma Physics of the CAS, Za Slovankou 3, 18200 Prague 8, Czech Republic \\ ${ }^{4}$ Max-Planck-Institut für Plasmaphysik, D-85748 Garching, Germany \\ ${ }^{5}$ York Plasma Institute, Department of Physics, University of York, York, YO10 5DD, UK
}

\begin{abstract}
The self-consistent core-pedestal prediction model of a combination of EPED1 type pedestal prediction and a simple stiff core transport model is able to predict type I ELMy pedestals of a large JET-ILW (ITER-like wall) database at the similar accuracy as is obtained when the experimental global plasma $\beta$ is used as input. The neutral penetration model [1] with corrections that take into account variations due to gas fuelling and plasma triangularity, is able to predict the pedestal density with an average error of $15 \%$. The prediction of the pedestal pressure in hydrogen plasma that has higher core heat diffusivity compared to a deuterium plasma with similar heating and fuelling agrees with the experiment when the isotope effect on the stability, the increased diffusivity and outward radial shift of the pedestal are included in the prediction. However, the neutral penetration model that successfully predicts the deuterium pedestal densities fails to predict the isotope effect on the pedestal density in hydrogen plasmas.
\end{abstract}

\section{Introduction}

In tokamaks with high-confinement (H-mode) operation steep density and temperature gradients develop near the plasma edge. In the region of steep gradients called the pedestal the turbulence that dominates the transport elsewhere in the plasma is greatly suppressed. In H-modes without an internal transport barrier, the heat transport is generally "stiff" in the core, due to turbulence increasing rapidly when the normalised temperature gradient $R / L_{T}(=R \nabla \mathrm{T} / \mathrm{T})$ increases beyond the critical value for turbulence onset. In these cases, the height of the temperature pedestal plays an important role in determining the confinement in the entire plasma. Consequently, predicting the pedestal becomes essential in predicting the performance of future experiments such as the coming DT campaign in JET.

While empirical scalings exists for the pedestal height [2,3], a physics-based model is required to be able to predict beyond the current experiments. One such model is the EPED model [4,5] that combines the pedestal peeling-ballooning mode (PBM) stability that is found to ultimately limit the pedestals in Type I ELMy H-mode discharges by acting as a trigger for ELMs and the kinetic ballooning mode (KBM) constraint that is assumed to limit the pedestal pressure gradient by increasing turbulence when the stability limit is reached.

However, the EPED model has inputs which include not only engineering parameters, ie. parameters that are known before the experiment, but also contains two parameters, total plasma $\beta$ and pedestal *See the author list of "Overview of the JET preparation for Deuterium-Tritium Operation" by E. Joffrin et al. to be published in Nuclear Fusion Special issue: overview and summary reports from the 27th Fusion Energy Conference (Ahmedabad, India, 22-27 October 2018) 
density $\mathrm{n}_{\mathrm{e}, \mathrm{ped}}$, that are not known in advance. In particular, $\beta$ depends on the plasma confinement, which in turn depends on the pedestal. In a fully predictive model these should be replaced by engineering quantities, such as neutral gas rate and heating power. Currently, the greatest challenge is the prediction of the pedestal density.

Furthermore, while most experiments use deuterium as fuel, future fusion devices such as ITER will be operated with deuterium-tritium and, with hydrogen and helium in the non-nuclear phase. The effect of the isotope mass on the plasma performance has been shown experimentally for JT-60U [6,7] ASDEX Upgrade [8], DIII-D [9] and JET (both with Carbon and Tungsten-Beryllium wall) $[10,11]$. The hydrogen plasmas need more heating power to reach the same plasma energy as in deuterium. In JET the difference in confinement is found to originate from the pedestal $[10,11]$. The EPED model has no explicit isotope dependency that can account to the observed difference.

In this paper, we will investigate how well the experimental observations can be reproduced with simple models for the pedestal density and core transport and how the changes in the details of the pedestal structure can reproduce the observed isotope effect.

\section{Peeling-ballooning stability of the JET pedestal database}

In this work we use the JET-ILW pedestal database that comprises 1072 stationary H-mode profiles fitted to the high-resolution Thomson scattering $[12,13]$ measurements taken at the last $30 \%$ of the ELM cycle with multiple measurements combined into a single fit[3]. The global parameter range of the discharges is for plasma current $\mathrm{I}_{\mathrm{p}}=1.0-4.0 \mathrm{MA}$, toroidal magnetic field $\mathrm{B}_{\mathrm{t}}=1.0-3.0 \mathrm{~T}$, total heating power $\mathrm{P}=3.4-33.1 \mathrm{MW}$ and plasma triangularity $\delta=0.18-0.45$. The variation in other plasma shape parameters is small: elongation $\kappa=1.6-1.8$, major radius $\mathrm{R}_{\mathrm{geo}}=2.81-2.97 \mathrm{~m}$ and minor radius $\mathrm{a}=0.83$ $0.96 \mathrm{~m}$. The strike-point location on the divertor is varied within the dataset. While experimentally it is found to affect the performance [14], it has no direct effect on the plasma stability as we model only the plasma inside the separatrix. The database includes a few cases with seeded impurities, $\mathrm{He}, \mathrm{N}$ and Ne. In the analysis we have omitted the discharges with pellets, vertical kicks or RMP ELM mitigation, as these ELM control methods are likely to affect the pedestal profiles through mechanisms not included in the model. This leaves 988 profiles for the comparison of predictive model results. In the analysis we assume that the ion temperature is equal to the electron temperature in the pedestal, and use the measured $Z_{\text {eff }}$ and appropriate dominant impurity (Beryllium in all other cases than impurity seeding, in which case the seeded impurity is used) to calculate ion density. Unless otherwise mentioned, the experimental profiles are radially shifted so that $T_{\mathrm{e}, \mathrm{sep}}=100 \mathrm{eV}$, which corresponds well to the power balance calculated using formulas in [15]. $\mathrm{T}_{\mathrm{e}, \mathrm{sep}}=100 \mathrm{eV}$ has also been assumed in all the predictive modelling. The radial shift is necessary due to the uncertainty in the location of the separatrix. Since both the density and temperature profiles come from the same diagnostic, the relative shift between the temperature and density pedestal positions is very well known and is taken into account in the analysis.

Before trying to predict the pedestals, we test how the assumption in the EPED model applies to the JET-ILW pedestal database. The EPED model assumes that the pedestal is ultimately limited by the peeling-ballooning stability. We have investigated this assumption by using the experimental density and temperature profiles in the HELENA equilibrium code [16] with an assumption that the current is a combination of fully diffused inductive and self-consistently calculated bootstrap current (see more details of the method in [17]). Then we have varied the temperature pedestal height, calculated new self-consistent equilibria for each profile and tested the stability of the equilibria using the ideal MHD stability code ELITE $[18,19]$. Any possible rotation shear effects on the stability are ignored as we do 
not have the accurate rotation measurements for the database. As a metric of distance of the experimental point to the stability boundary we have used $\alpha_{c r i t} / \alpha_{\text {exp }}$, where $\alpha_{\text {crit }}$ and $\alpha_{\text {exp }}$ refer to the values of normalised pressure gradient $\alpha$ at the stability boundary following the self-consistent path and of the experimental profile, respectively. The stability boundary is defined as $\gamma>0.25 \omega^{*}{ }_{\text {max }}$, where $\gamma$ is the growth rate of the fastest growing mode and $\omega^{*}$ max is the maximum of the ion diamagnetic frequency in the pedestal region. The distance to the stability boundary as a function of the experimental normalised $\beta_{\mathrm{N}}$ is plotted in Fig. 1 . We find that only about $40 \%$ of the analysed pedestals can be considered to be limited by the PBMs at the end of the ELM cycle. The remaining fraction is found to be stable for PBMs. At high $\beta_{\mathrm{N}}$ the proportion of PBM limited cases is higher being about $71 \%$ for $\beta_{\mathrm{N}}>2.5$. Using a different criterion for stability (such as $\gamma>0.03 \omega_{\mathrm{A}}$, where $\omega_{\mathrm{A}}$ is the Alfvén frequency) changes the $\alpha_{c r i t} / \alpha_{\text {exp }}$ ratio in some cases but has little effect on the general result.

Sheared rotation and $\mathrm{T}_{\mathrm{i}}>\mathrm{T}_{\mathrm{e}}$ in the pedestal region have been found to lower the ballooning stability limit in JET [20]. However, we do not have the $\mathrm{T}_{\mathrm{i}}$ or rotation profiles available for the large database investigated here. Therefore, these effects were ignored in the analysis. It is possible that they explain at least some of the cases that were found to be far from the stability boundary.

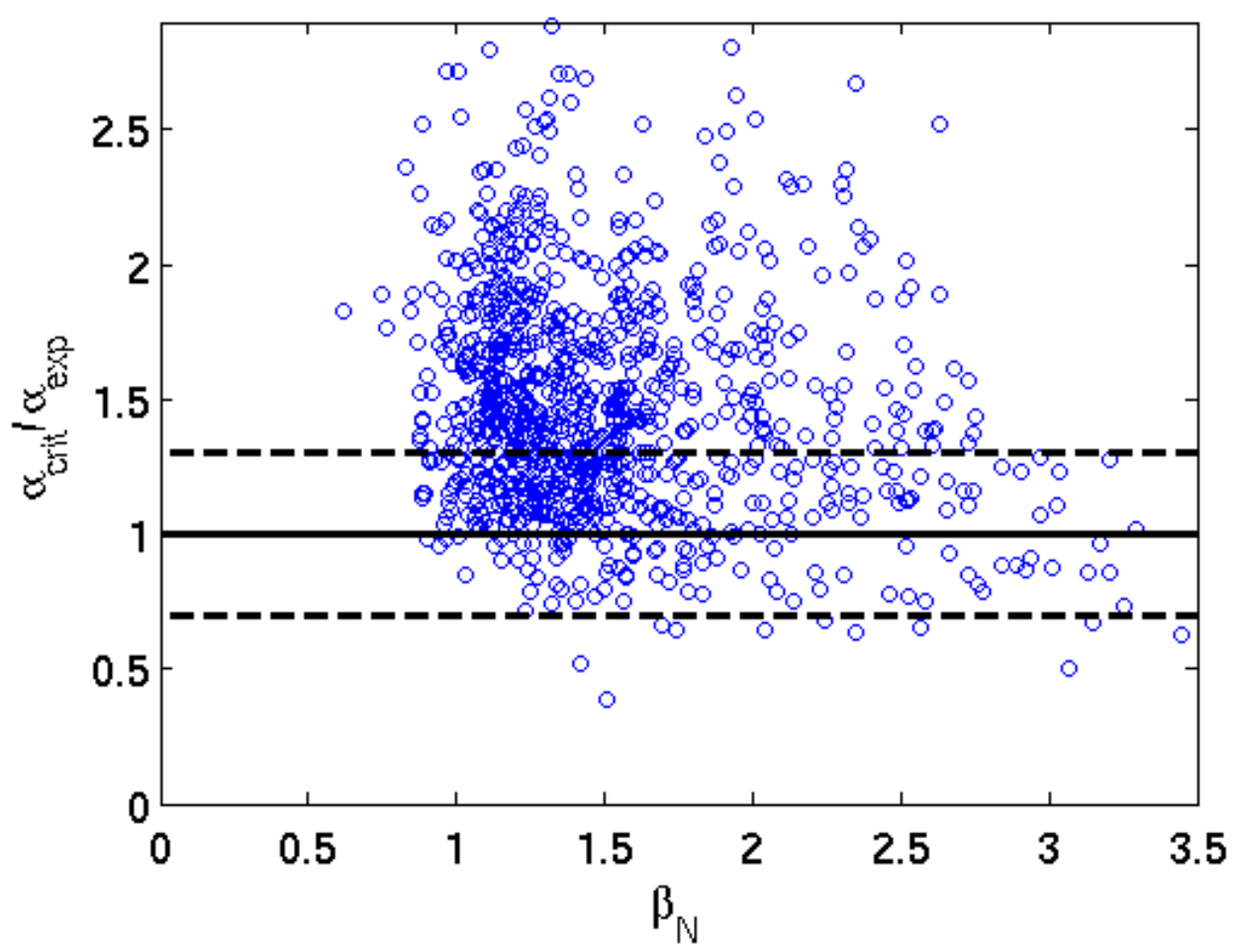

Figure 1. The ratio $\alpha_{c r i t} / \alpha_{\text {exp }}$ of the pedestal database plotted as a function of $\beta_{\mathrm{N}}$. The solid line represents the situation where the experimental profile is exactly on the peeling-ballooning stability boundary. The dashed lines represent $30 \%$ error margins and the points between them are considered to be limited by peeling-ballooning modes at the end of the ELM cycle.

We conduct the standard EPED1 model (the same parameterisation for the core profiles as in [3]) predictions for the entire database and find that the EPED1 model predicts the pedestal pressure for the entire database with an average error of $11 \%$ (RMSE $=14 \%$, with RMSE or root mean square of error defined as $\sqrt{\sum_{i=1}^{n}\left(p_{e, p r e d}-p_{e, e x p}\right)^{2} / n}$, where $\mathrm{n}$ is the total number of cases). If we restrict the 
analysis only to the cases with $\alpha_{\text {crit }} / \alpha_{\text {exp }}<1.3$, the average error of predicted pedestal pressure is reduced to $10 \%$ (RMSE $=13 \%$ ). As can be seen in Fig 2 the experimental pedestals that are not close to the PBM stability boundary tend to be over-predicted with the EPED1 model, while the pedestal that are close to the stability boundary, are mainly under-predicted. The almost identical accuracy of the prediction for the cases whose experimental pedestals are near and far from the PBM stability boundary is a result of the pedestal width being under-predicted in the cases that are not near the PBM boundary. The prediction for the pedestal width is shown in Fig. 3. The average error for the width (defined as in [4] to be the average of the density and temperature pedestal widths) is $21 \%$ for the cases with $\alpha_{\text {crit }} / \alpha_{\text {exp }}<1.3$ and $34 \%$ for the cases with $\alpha_{\text {crit }} / \alpha_{\exp }>1.3$. As the experimental density and temperature profiles are not aligned, we also compare predicted width to the fitted pressure profile width. This improves the accuracy of the width prediction with average error of $14 \%$ for the for the cases with $\alpha_{\text {crit }} / \alpha_{\exp }<1.3$ and $23 \%$ for the cases with $\alpha_{\text {crit }} / \alpha_{\exp }>1.3$. As the focus of this paper is not the validation of the EPED1 model itself, in the further analysis we restrict the database only to the experimental cases that are close to the stability boundary, since we can then expect the basic EPED1 assumptions to be better fulfilled than in the entire database.

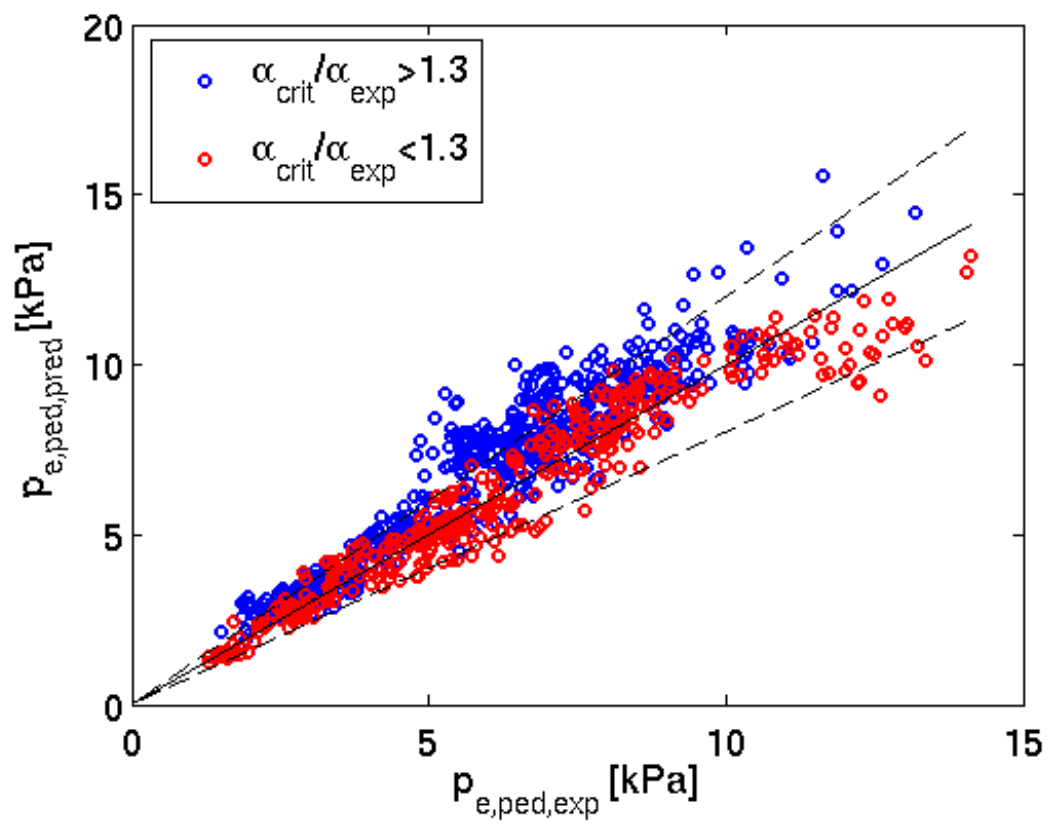

Figure 2. The predicted pedestal top electron pressure against the experimental electron pedestal top pressure for the cases close to the PBM stability limit $\left(\alpha_{\text {crit }} / \alpha_{\text {exp }}<1.3\right.$, red $)$ and far from the stability limit $\left(\alpha_{\text {crit }} / \alpha_{\text {exp }}>1.3\right.$, blue). The solid line marks the perfect match and the dashed lines represent $\pm 20 \%$ error. 

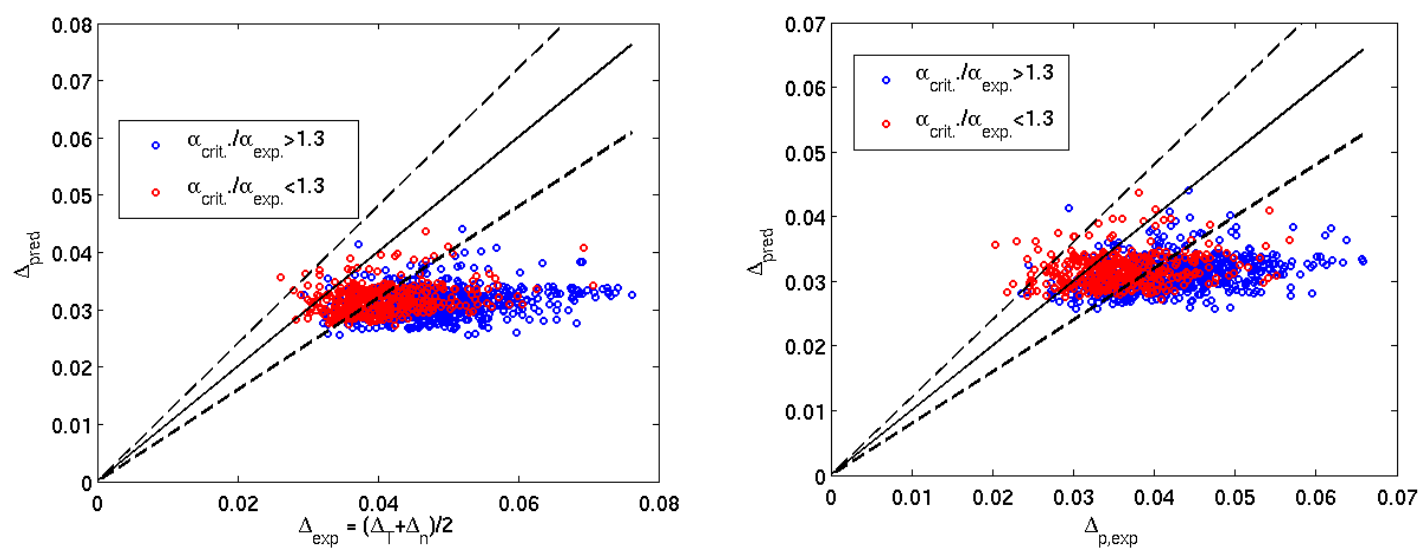

Figure 3. The predicted pedestal width against the experimental electron pedestal width, (define as $\Delta=\left(\Delta_{\mathrm{Te}}+\Delta_{\mathrm{ne}}\right) / 2$,left and fitted pressure width, right) for the cases close to the PBM stability limit $\left(\alpha_{\text {crit }} / \alpha_{\text {exp }}<1.3\right.$, red $)$ and far from the stability limit $\left(\alpha_{\text {crit }} / \alpha_{\text {exp }}>1.3\right.$, blue $)$. The solid line marks the perfect match and the dashed lines represent $\pm 20 \%$ error.

\section{Pedestal density prediction}

The EPED1 model used above takes the pedestal density as known in advance and temperature as predicted. This is valid assumption if the density can be fully controlled with some actuators such as gas fuelling or pellets. In such a case there is no need to predict the pedestal density prior to the experiment as the desired density can be achieved by using these actuators. However, in JET-ILW the gas fuelling has been found to have a relatively minor effect on the resulting density, with the gas rate variation from 0.2 to $3.8 \times 10^{22} \mathrm{el} / \mathrm{s}$ having very little effect on the pedestal density height in deuterium [21]. Additionally, there may be requirements on the fuelling to protect the divertor from excessive heat loads, which may not allow the gas rate to be used as a control actuator for the pedestal density. Therefore, the pedestal prediction model should predict the density together with the temperature. Here we present models that can be used to predict density in JET-ILW pedestals and the testing of them against the measured data.

The pedestal density is controlled by several processes. The neutral gas in the scrape-off layer and the pellets act as a source. The neutral particles deposited in the core plasma by the neutral beams are transported into the pedestal. The turbulence and collisional transport move particles in (pinch) and out (diffusion) of the pedestal between ELMs and finally the ELMs cause rapid loss of particles from the pedestal. Modelling all the processes would require time dependent turbulence simulations of the pedestal and the scrape-off layer along with a non-linear ELM model, which is beyond the scope of this paper. As a first step we use much more simplified methods to predict the density pedestal in JET-ILW.

Urano [22] parameterised the experimental JET-ILW density pedestal data. The parameters used were plasma current ( $\mathrm{I}_{\mathrm{p}}$ in MA), vacuum toroidal magnetic field $\left(\mathrm{B}_{\mathrm{t}}\right.$ in $\left.\mathrm{T}\right)$, plasma triangularity $(\delta)$, neutral beam heating power $\left(\mathrm{P}_{\mathrm{NBI}}\right.$ in $\left.\mathrm{MW}\right)$ and gas fuelling rate $\left(\phi\right.$ in $\left.10^{22} \mathrm{e} / \mathrm{s}\right)$. All the parameters are known in advance of the experiment and can be used in the prediction. The parameterisation is the following:

$$
n_{e, p e d}\left[10^{19} m^{-3}\right]=8.05 \cdot I_{p}^{1.28} \cdot B_{t}^{-0.6} \cdot P_{N B I}{ }^{-0.07} \cdot \delta^{0.54} \cdot \Phi_{e}{ }^{0.10} .
$$


The parameterisation does not contain any physics understanding of the processes controlling the density but can be used in a prediction model as long as it is used in the same parameter range that was used in the fitting of the parameters.

In a second, more physics-based approach we have used the neutral penetration model (NPM) [1]. This model assumes that all the fuelling is from the plasma edge and that the particle diffusion coefficient $\mathrm{D}$ is constant in space and leads to a relation between the width $\Delta_{\mathrm{ne}}$ in real units on the midplane and height $\mathrm{n}_{\mathrm{e}, \mathrm{ped}}$ of the pedestal:

$$
\Delta_{n e}=2 V_{n} /\left(\sigma_{i} V_{e} E n_{e, p e d}\right) .
$$

Here $V_{n}$ is the velocity of the neutral particles, $\sigma_{\mathrm{i}}$ is the cross section for electron impact ionization, $V_{\mathrm{e}}$ is the electron thermal velocity at the top of the pedestal and $\mathrm{E}$ represents the flux expansion factor between the location of fuelling and the midplane. $V_{\mathrm{n}}$ can be calculated assuming that we know the pedestal temperature by taking into account Frank-Condon and charge-exchange neutrals in the formulas [23]:

$$
\begin{gathered}
v_{C E}=\sqrt{\frac{T_{i}}{2 \pi m_{i}}} \\
v_{F C}=\frac{2}{\pi} \sqrt{\frac{3}{m_{i}}} \\
w=\frac{\frac{v_{F C}}{2} \sigma_{i} V_{e}}{\left(v_{F C} \sigma_{i} V_{e}-v_{C E} \sigma_{i} V_{e}-v_{C E} \sigma_{C E} V_{e}\right)} \\
v^{*}=\frac{\left(v_{F C} \sigma_{i} V_{e}+\frac{v_{C E} \sigma_{C E} V_{e}}{2}\right)}{\left(\sigma_{i} V_{e}+\frac{\sigma_{C E} V_{e}}{2}\right)} \\
V_{n}=\frac{v_{F C} w+v^{*}}{w+1},
\end{gathered}
$$

where $\sigma_{i} V_{e}$ is the ionization rate coefficient, $\sigma_{C E} V_{e}$ the charge exchange rate coefficient, $V_{\mathrm{FC}}$ the velocity of the Frank-Condon neutrals, $V_{\mathrm{CE}}$ the velocity of the charge exchange neutrals and $w$ is the ratio of charge exchange to Frank-Condon neutrals reaching the separatrix. The ionisation and charge exchange rate coefficients are calculated using the pedestal temperature and the rates as a function of particle energy in [24].

For each hypothetical pedestal width, we assume that $\Delta=\Delta_{\mathrm{ne}}=\Delta_{\mathrm{Te}}$ and use the EPED1 constraint $\Delta=$ $0.076 \sqrt{\beta_{p, p e d}}$ to calculate the pedestal pressure height (from $\beta_{p, p e d}$ ). Using an initial guess for the density, the temperature (assuming $T_{i}=T_{e}$ and known effective charge $Z_{\mathrm{eff}}$ ) is calculated from the pressure. The temperature and the pedestal width is used to calculate the new pedestal density using the formulas (2-7). A new temperature is calculated from the pressure and density and the process is continued until it converges. Note that when combined with the EPED1 model, the final resulting density corresponds to the pedestal width of the equilibrium that is at the PBM stability limit.

As the flux expansion factor $\mathrm{E}$ is the only free parameter in the model, we first use a constant factor $\mathrm{E}=5$ based on the MAST results [23]. This assumes all the neutral particles enter the plasma through the X-point. Figure 4 shows how the parameterised density and the neutral penetration model combined with the EPED1 model for the rest of the prediction match the experimental density of the PBM limited cases. The average error for the parameterised density is $12 \%(\mathrm{RMSE}=16 \%)$ and for the 
neutral penetration model 19\% (RMSE=23\%). Note that both the parameterised density and the neutral penetration model flatten out at the highest experimental densities leading to underprediction there.

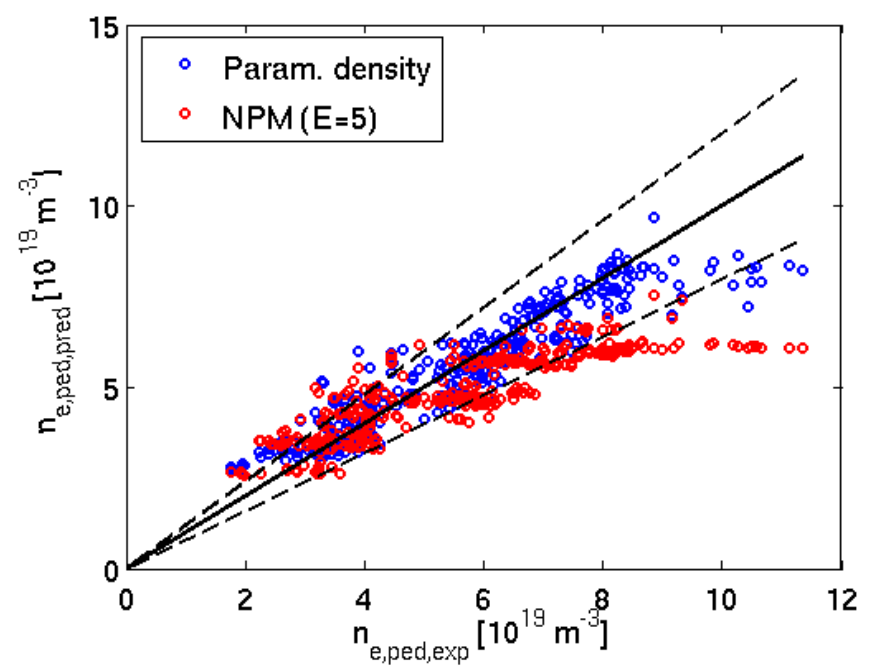

Figure 4. The predicted pedestal density against the experimental pedestal density for the parameterised density and neutral penetration model with $\mathrm{E}=5$. The solid line marks the perfect match and the dashed lines represent $\pm 20 \%$ error.

To improve the neutral penetration model, we take into account that as the gas fuelling is increased more particles are likely to enter the confined plasma from other parts of the poloidal plane than just the X-point. This can be taken into account by making the flux expansion factor dependent on the gas fuelling rate. The average error in the neutral penetration model is reduced to $15 \%$ (RMSE=19\%) if we use $E=4.8 \phi^{-0.20}$, where $\phi$ is the gas fuelling rate in units of $10^{22}$ electrons/s. Note that here the gas rate is defined as the output of the gas valves. The actual neutral density in the scrape-off layer is affected also by the pumping efficiency which varies with the strike point position on the divertor. The parameters are set to give the best fit to the data. As can be seen in Fig. 5. the error in the neutral penetration model depends on the fuelling rate and correcting this improves the match with the experiment. Note that the correction term exponent is relatively small indicating only a weak dependence of pedestal density to the fuelling rate.
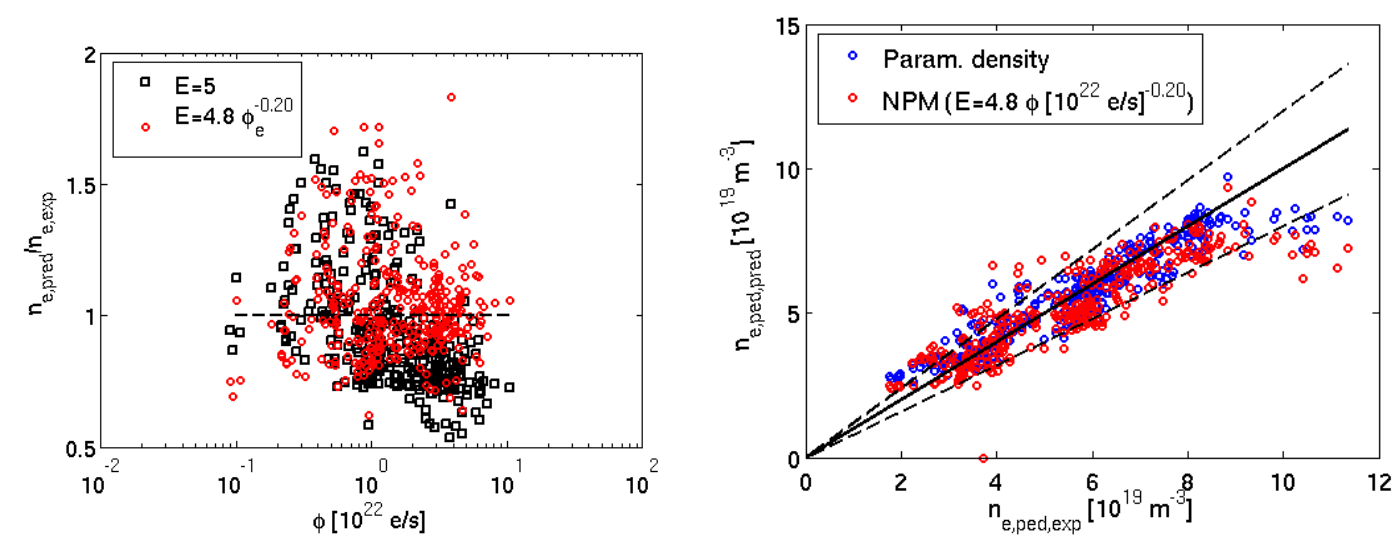
Figure 5. The ratio of predicted density with the neutral penetration model with $E=5$ (black) and $E=$ $4.8 \phi^{-0.20}$ (red) to the experimental density as a function of the gas fuelling rate (left) and the predicted density with the neutral penetration model with a correction for the fuelling rate against the experiment (right). The solid line marks the perfect match and the dashed lines represent $\pm 20 \%$ error.

However, we can see that at the highest densities the model still under-predicts the experiment. If we plot the ratio of the predicted density to the experimental density against the average plasma triangularity (Fig 6, left), we can see clearly that the experimental density increase with triangularity is not captured by the model. The density dependency on the plasma triangularity is in the parameterisation by Urano and has also been observed in ASDEX Upgrade [8]. The neutral penetration model itself does not have a physical explanation for the higher density at high triangularity. However, if we include the triangularity effect ad hoc on the flux expansion factor ( $E=$ $2.4 \delta^{-0.53} \phi^{-0.20}$, where $\delta$ is the triangularity), the model is able to predict the density with an average error of $13 \%$ (RMSE $=17 \%$ ), which is very close to the value of the parameterised density error. It must be noted, however, that while a positive dependency of density with triangularity has also been observed for instance in JET-C (JET with a carbon wall) [22] the exact triangularity power dependency in the model may be JET-ILW specific and other devices may have different dependency on the plasma shape. The predicted density using the final neutral penetration model and the parameterised density against the experimental density are shown in Fig 6 (right) showing very similar match. The possible physical mechanisms for the triangularity dependency are that the interELM particle turbulent transport is affected by the triangularity, the particle flux due to ELMs is affected by triangularity (the ELM frequency decreases with triangularity when all other parameters are kept fixed) and that at high triangularity, the recycling from the top of the device increases. The last mechanism is JET specific as it is difficult to make high triangularity plasma with large clearance from the top or without bringing the second X-point inside the wall. Note that the neutral penetration model predicts the density self-consistently with the pedestal width, ie. no assumption of the pedestal width is made prior to the prediction except that it follows the EPED1 scaling with the $\beta_{\mathrm{p}, \mathrm{ped}}$ and that density and temperature pedestal widths are equal. All the dependencies of the plasma current and the magnetic field, which are the strongest coefficients in the density parameterisation, are reproduced by the model without explicitly depending on them.
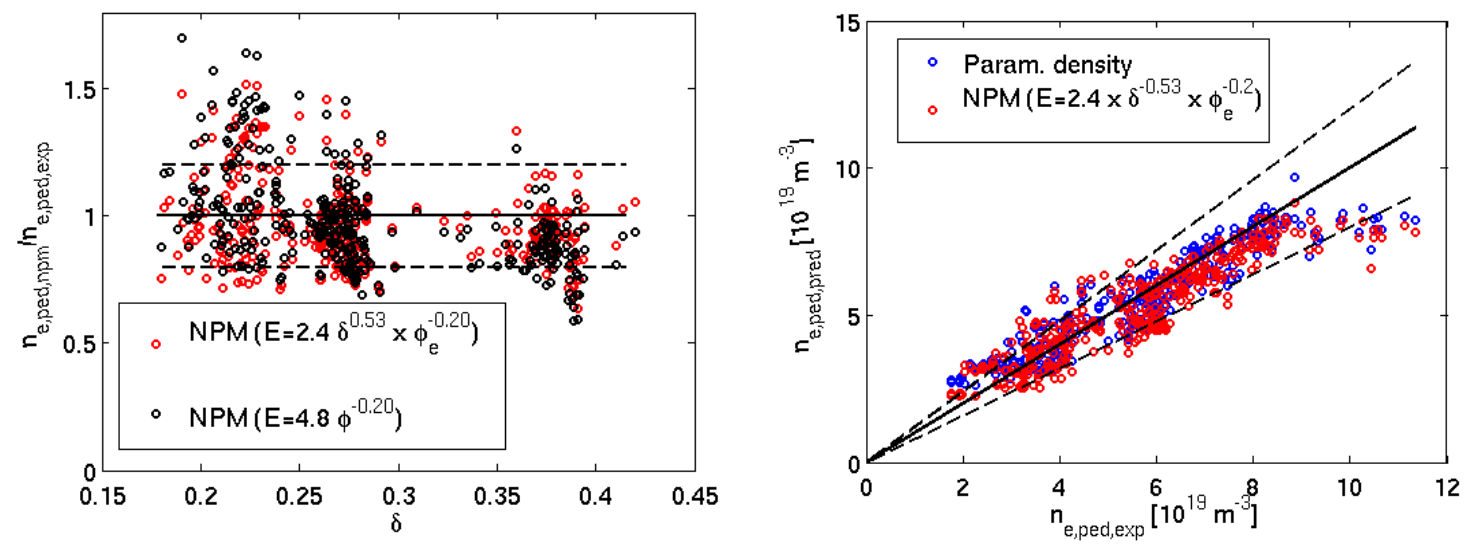

Figure 6. The ratio of predicted and experimental density for the neutral penetration model (left) with $E=4.8 \phi^{-0.20}$ (black) and $E=2.4 \delta^{-0.53} \phi^{-0.20}$ (red). The solid line represents a perfect prediction and the dashed lines show the $\pm 20 \%$ error. The predicted density with the neutral penetration model with a correction for the fuelling rate and plasma triangularity against the experiment (right). The solid line marks the perfect match and the dashed lines represent $\pm 20 \%$ error. 


\section{Self-consistent core-pedestal modelling}

In an H-mode tokamak plasma the core $\beta_{\mathrm{p}}\left(=2 \mu_{0}\langle p\rangle /\left\langle B_{p}^{2}\right\rangle\right)$ affects the MHD stability of the edge $[25,26]$. At the same time the pedestal sets the boundary condition for the core transport. For predicting the exact core plasma profile shapes the turbulent flux in the core should be modelled with sophisticated non-linear gyrokinetic codes or quasi-linear codes that use saturation rules based on the non-linear runs such as TGLF [27]. In ITER simulations using TGLF, it was found that the fusion power in the core scaled as $p_{\text {ped }}^{2}$ [28] indicating a very stiff transport in the core. TGLF together with EPED1 was also used in optimisation of ITER scenarios in terms of density and $Z_{\text {eff }}$ [29].

The transport models based on non-linear or quasi-linear gyrokinetic codes can be slow to converge. If we are not interested in solving the exact core plasma profile shapes, but still want to solve the pedestal accurately taking into account the stabilisation by the core, we can rely on the fact that the core temperature profiles tend to be stiff to give a fast model for the core transport. We simulate the steady state core heat transport of electrons (e) and ions (i) with a simple model:

$$
\frac{\partial T_{e, i}}{\partial \varrho}=-\frac{q_{e, i}}{V \prime\left\langle|\nabla \varrho|^{2}\right\rangle n_{e, i} \chi_{e, i}},
$$

where $\chi$ is the diffusivity, $\mathrm{V}^{\prime}=\mathrm{dV} / \mathrm{d} \rho$ is the radial derivative of the plasma volume, $\mathrm{n}$ is the density, $\mathrm{q}_{\mathrm{e}}$ is the heat flux and $\rho$ is a radial coordinate. For the heating profile, we use a simple form $\mathrm{P} / \mathrm{V}\left(\psi_{\mathrm{N}}\right)=\mathrm{P}_{\mathrm{c}}\left(\mathrm{w}_{\mathrm{h}}-\psi_{\mathrm{N}}\right)$, when $\psi_{\mathrm{N}}<\mathrm{w}_{\mathrm{h}}$ and 0 when $\psi_{\mathrm{N}}>\mathrm{w}_{\mathrm{h}}$, where $\mathrm{w}_{\mathrm{h}}$ is the width of the heating region. $\mathrm{P}_{\mathrm{c}}$ is adjusted so that the volume integrated power matches that of the experiment. We choose $\mathrm{w}_{\mathrm{h}}=0.3$ but note that the results are not very sensitive to the choice of the shape and width of the heating profile. At each flux surface, the heat flux through the surface, $q_{\mathrm{e}, \mathrm{i}}$ is calculated from the heating profile and the surface area of that surface. For the diffusivity $\chi$ we use a simple model that produces stiff temperature profiles:

$\chi_{i, e}=C 1$, when $\frac{R \nabla T}{T}<\left(\frac{R \nabla T}{T}\right)_{\text {crit }}$

$\chi_{i, e}=C 1+C 2\left[\frac{R \nabla T}{T}-\left(\frac{R \nabla T}{T}\right)_{c r i t}\right]$, when $\frac{R \nabla T}{T}>\left(\frac{R \nabla T}{T}\right)_{c r i t}$,

where $\mathrm{C} 1, \mathrm{C} 2$ and $(R \nabla T / T)_{\text {crit }}$ are free parameters. To produce a stiff heat transport model, $\mathrm{C} 1$ is set to a much lower value than $\mathrm{C} 2$. We choose $(R \nabla T / T)_{c r i t}=5.0$ based on both experimental JET results [30] and gyrokinetic simulation [30,31]. $\mathrm{C} 1$ is set to $0.1 \mathrm{~m}^{2} / \mathrm{s}$ and the results are not very sensitive to this value as long as it is much smaller than C2. The results for two values of $\mathrm{C} 2: 1 \mathrm{~m}^{2} / \mathrm{s}$ and $2 \mathrm{~m}^{2} / \mathrm{s}$ are shown in Fig. 7 and 8 . As can be seen both values of $\mathrm{C} 2$ have most of the points within the $20 \%$ error margins. The average error of the pedestal pressure height prediction for both values of $\mathrm{C} 2$ is slightly higher at $15 \%\left(\mathrm{RMSE}=19 \%\right.$ for $\mathrm{C} 2=1 \mathrm{~m}^{2} / \mathrm{s}$ and $\mathrm{RMSE}=17 \%$ for $\mathrm{C}=2 \mathrm{~m}^{2} / \mathrm{s}$ ) than for the standard EPED1 predictions with the known value of $\beta$. Interestingly the electron temperature in the core (defined at $\psi=0.2$ as the Thomson scattering measurement does not go through the magnetic axis) is actually predicted better with the self-consistent model with $\mathrm{C}=2 \mathrm{~m}^{2} / \mathrm{s}$ than with the standard EPED1. The most likely reason for that is that in EPED1 model, we assume $T_{i}=T_{e}$ in the core and the core temperature profiles are adjusted to match the experimental $\beta_{p}$. At low collisionality $T_{i}>T_{e}$ in the experiment but this is not reflected in the modelling. If the temperatures were allowed to separate in the core at low collisionality, the core $\mathrm{T}_{\mathrm{e}}$ would decrease in these cases. With $\mathrm{C} 2=1 \mathrm{~m}^{2} / \mathrm{s}$ the behaviour of the predicted core electron temperature is similar to the standard EPED1 model. The effect of 
changing $\mathrm{C} 2$ from $2 \mathrm{~m} 2 / \mathrm{s}$ to $1 \mathrm{~m} 2 / \mathrm{s}$ is an increase of $25 \% \pm 5 \%$ in core temperature and $10 \% \pm 5 \%$ in pedestal pressure.
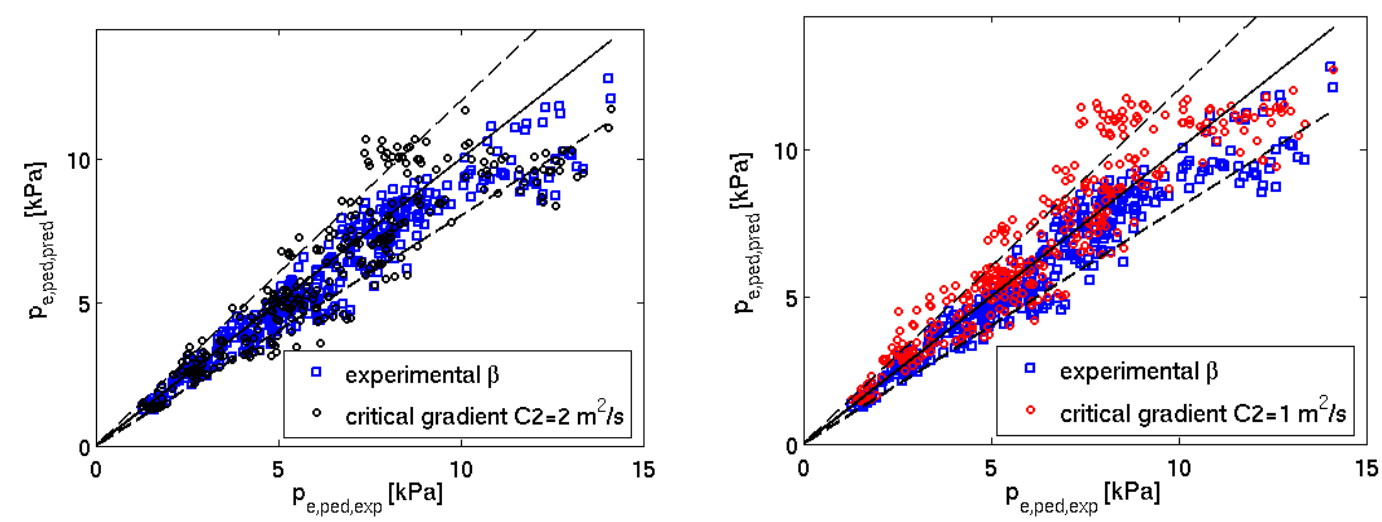

Figure 7. The predicted pedestal pressure for the two values of C2 $\left(2 \mathrm{~m}^{2} / \mathrm{s} \mathrm{black,} \mathrm{left,} 1 \mathrm{~m}^{2} / \mathrm{s} \mathrm{red}\right.$, right) in the transport model (red circles) and standard EPED1 mode (blue circles). The solid line represents a perfect prediction and the dashed lines show the $\pm 20 \%$ error.
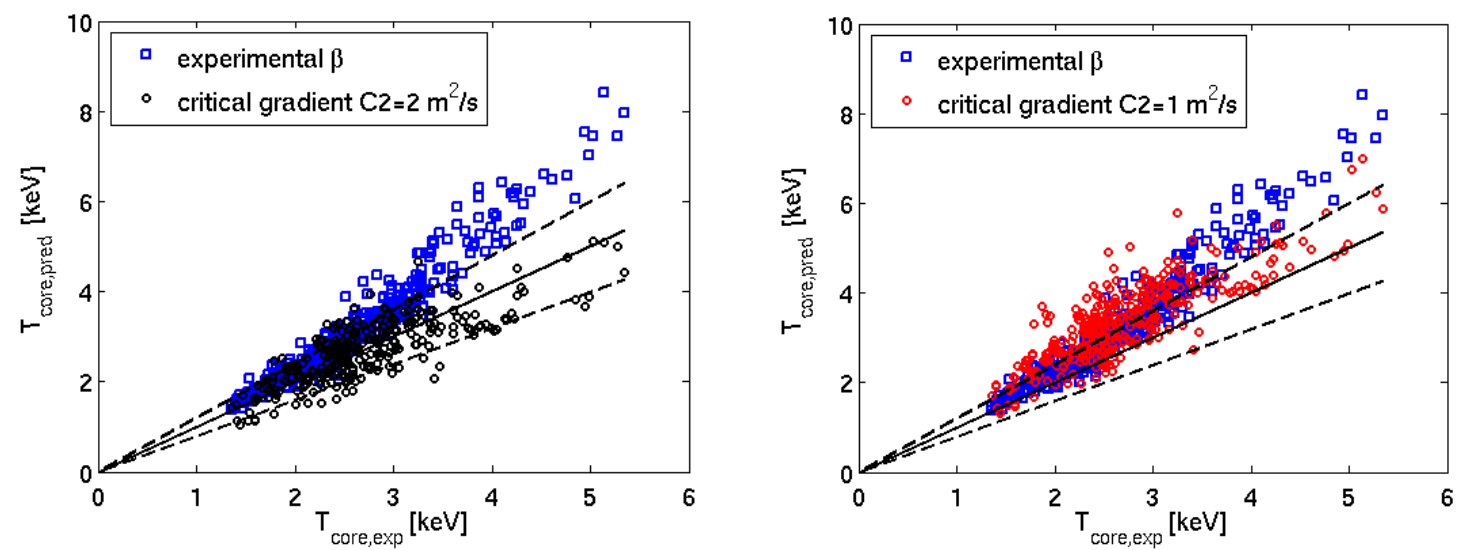

Figure 8 . The predicted core $\left(\psi_{\mathrm{N}}=0.2\right)$ temperature for the two values of $\mathrm{C} 2\left(2 \mathrm{~m}^{2} / \mathrm{s} \mathrm{black}\right.$, left, $1 \mathrm{~m}^{2} / \mathrm{s}$ red, right) in the transport model (red circles) and standard EPED1 mode (blue circles). The solid line represents a perfect prediction and the dashed lines show the $\pm 20 \%$ error.

The core density profile cannot be assumed to be stiff like the temperature profile. We compute the core density peaking by using the formula from [33] and for simplicity discard the effect of neutral beam fuelling and increase the collisionality coefficient (the original coefficients were a fit to data in any case). The density peaking factor is then:

$$
n_{p k}=n\left(\psi_{N}^{0.5}=0.2\right) /\langle n\rangle=1.347-0.25 \ln \left(v_{e f f}\right)-4.03 \beta,
$$

where $v_{\text {eff }}$ is the effective collisionality and is defined as $v_{\text {eff }}=0.2\left\langle n_{\text {ped }}\right\rangle R_{0} /\left\langle T_{e}\right\rangle^{2}$ and $\beta=$ $4.02 \times 10^{-3}\langle p\rangle / B_{T 0}^{2}$, with $\langle p\rangle=2\langle T\rangle n_{p e d}$ (in units of $\mathrm{keV} \times 10^{19} \mathrm{~m}^{-3}$ ). Here the pedestal density is used as a proxy for the average density in the calculation of collisionality as the core density is the 
unknown. The experimental and predicted density peaking for the database are shown in Fig. 9. The general trend with collisionality is reproduced, but some of the variation in the experimental data is not captured. The effect of this is very small on the pedestal prediction with the average error and RMSE staying unchanged from the runs done with experimental density profiles.

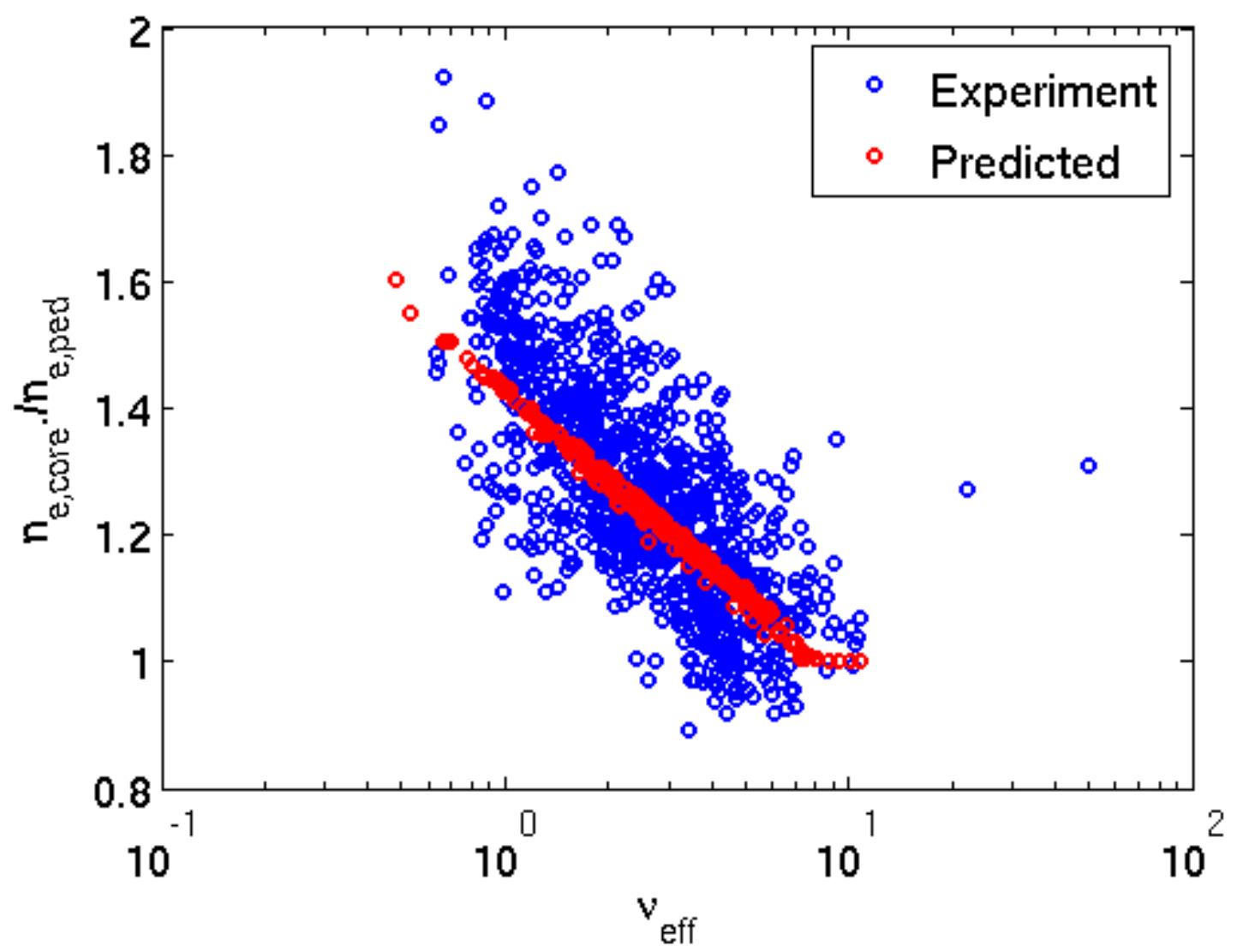

Figure 9. The predicted and experimental density peaking $\left(\mathrm{n}_{\mathrm{e}}\left(\psi_{\mathrm{N}}=0.2\right) / \mathrm{n}_{\mathrm{e}, \mathrm{ped}}\right)$ as a function of effective collisionality.

We combine the neutral penetration model with the triangularity and fuelling rate corrections for the prediction of $n_{e, p e d}$, the core heat transport model (9)-(10) with parameters $C 2=2 \mathrm{~m}^{2} / \mathrm{s}$ and $\mathrm{C} 1=0.1 \mathrm{~m}^{2} / \mathrm{s}$ and the core density peaking formula (11) for the self-consistent prediction of the pedestal and core. The only assumptions about the profiles left in this model is the value of temperature at the separatrix $(=100 \mathrm{eV}$, as used in the stability calculations $)$ and the ratio of separatrix to pedestal density $\mathrm{n}_{\mathrm{e}, \mathrm{sep}} / \mathrm{n}_{\mathrm{e}, \mathrm{ped}}$ $\left(=0.25\right.$, as used in EPED1 model). Increasing $n_{e, s e p} / n_{e, p e d}$ to 0.5 (which is the average for this database) increases the pedestal top pressure prediction by about $5 \%$ due to the decrease of the pedestal pressure gradient. The experimental value of $Z_{\text {eff }}$ is used as we have no model for the prediction of the impurity content. The result of the self-consistent pedestal prediction compared to the standard EPED1 model prediction with the prior knowledge of $\beta$ and $\mathrm{n}_{\mathrm{e}, \text { ped }}$ is shown in Fig 10. The average error of the fully self-consistent prediction is $13 \%$ and $\mathrm{RMSE}=16 \%$. 


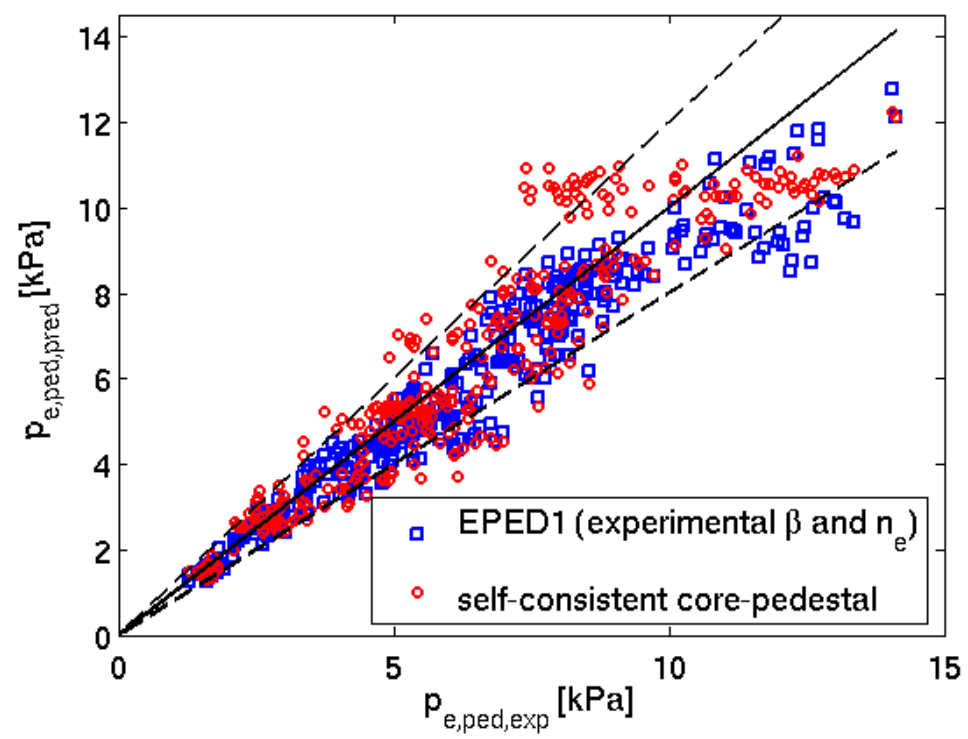

Figure 10. The predicted pedestal pressure for the EPED1 model with experimental $\beta$ and core and pedestal density in input (blue) and the self-consistent core-pedestal model (red) against the experimental pedestal pressure. The solid line represents a perfect prediction and the dashed lines show the $\pm 20 \%$ error.

\section{The isotope effect in pedestal prediction}

The pedestal confinement in JET is known to scale positively with the isotope mass of the fuel ions $[10,11,34,35]$. Here we investigate how the pedestal prediction model can include the isotope effect by comparing the predictions for three Type I ELMy JET-ILW discharges (\#84793, \#84796 and $\# 91554$ ) at $\mathrm{I}_{\mathrm{p}}=1.4 \mathrm{MA}, \mathrm{B}_{\mathrm{t}}=1.7 \mathrm{~T}$. The gas rate is the same for all the discharges. Two of the discharges (\#84793 and \#84796) are in deuterium and one (\#91554) in hydrogen. One of the deuterium discharges (\#84793) matches the hydrogen discharge in stored energy $\left(\mathrm{W}_{\mathrm{p}}=1.5 \mathrm{MJ}\right)$ but is achieved with lower heating power ( $\mathrm{P}=8.7 \mathrm{MW}$ in hydrogen and $5 \mathrm{MW}$ in deuterium). The other deuterium discharge (\#84796) has similar heating power (10MW) with the hydrogen discharge, but the stored energy in that discharge is higher $\left(\mathrm{W}_{\mathrm{p}}=2.5 \mathrm{MJ}\right)$. This means that in hydrogen the energy confinement is degraded compared to deuterium [11].

In the predictive pedestal modelling the isotope mass has a direct effect on the pedestal stability only through the Alfvén frequency that scales as $1 / \sqrt{\varrho}$, where $\rho$ is the mass density of the plasma. The growth rates of the MHD instabilities scale with Alfvén frequency and consequently with $1 / \sqrt{\varrho}$. For the same pressure and current profiles, the instability grows $\sqrt{2}$ times faster in the hydrogen plasma than in the deuterium plasma. As described in Sec 1. we use the criterion $\gamma>0.25 \omega^{*}{ }_{\text {max }}$ for the peelingballooning mode boundary. The diamagnetic frequency $\omega^{*}$ does not depend on the isotope mass, implying that the peeling-ballooning mode boundary shifts to a lower value of $\alpha$ in a hydrogen plasma compared to a deuterium plasma. However, this effect is relatively minor and cannot alone explain the effect of the isotope mass on the pedestal.

In the neutral penetration model of Sec. 3 the isotope mass affects only the velocity of neutrals, $V_{n}$ in (2) leading to higher predicted density in hydrogen than in deuterium if the marginally stable pedestal width does not change. However, the experimental behaviour is the opposite, ie. the pedestal density decreases in hydrogen compared to deuterium with the same gas fuelling rate $[11,37]$. This indicates 
that while we were able to get relatively good predictions in deuterium plasmas with the neutral penetration model, it alone is not able to explain the pedestal density in hydrogen. The inter-ELM particle transport as well as increased ELM frequency [11] in hydrogen plasmas must also play a role in setting the density pedestal. It is also possible that to accurately describe the physics of neutral fuelling, a more comprehensive model is required. As the neutral penetration model alone does not reproduce the correct density behaviour with isotope mass, we will use experimental densities in the following core-pedestal modelling.

In the self-consistent core-pedestal modelling we use the same transport model for the hydrogen plasma as for the deuterium cases but take into account that the core heat diffusivity is doubled for hydrogen compared to deuterium for the similar normalised temperature gradient [11]. The main reason for the higher diffusivity in hydrogen is the systematically lower density. The total heat transport in the core is similar with both isotopes.

Figure 11 shows the predicted pedestal pressure using the experimental density as input and the selfconsistent core-pedestal model with the experimental heating powers. As expected, if the same settings are used for all the three discharges, the predicted pedestal pressure increases with heating power with the hydrogen case prediction being slightly decreased by the direct isotope effect on the stability. That prediction is significantly higher than the experimental pedestal. However, when the higher core diffusivity is included into the prediction, the prediction for the hydrogen case is closer to the experimental case but it still slightly overpredicted. The global $\beta_{\mathrm{N}}$ values in the three cases are also relatively well predicted.

There is an indication [37] that the hydrogen H-modes may have a higher separatrix temperature than the deuterium plasmas. As we use the separatrix temperature as an anchor for radial position of the pedestal profiles with respect to the separatrix, the increased separatrix temperature would imply that the position of the maximum pressure gradient is closer to the separatrix, which in turn would have a degrading effect for the pedestal stability against the ballooning modes. In this particular case, the separatrix temperature would have to be $150 \mathrm{eV}$ or higher to achieve similar accuracy for the hydrogen case prediction as is obtained for the deuterium cases.
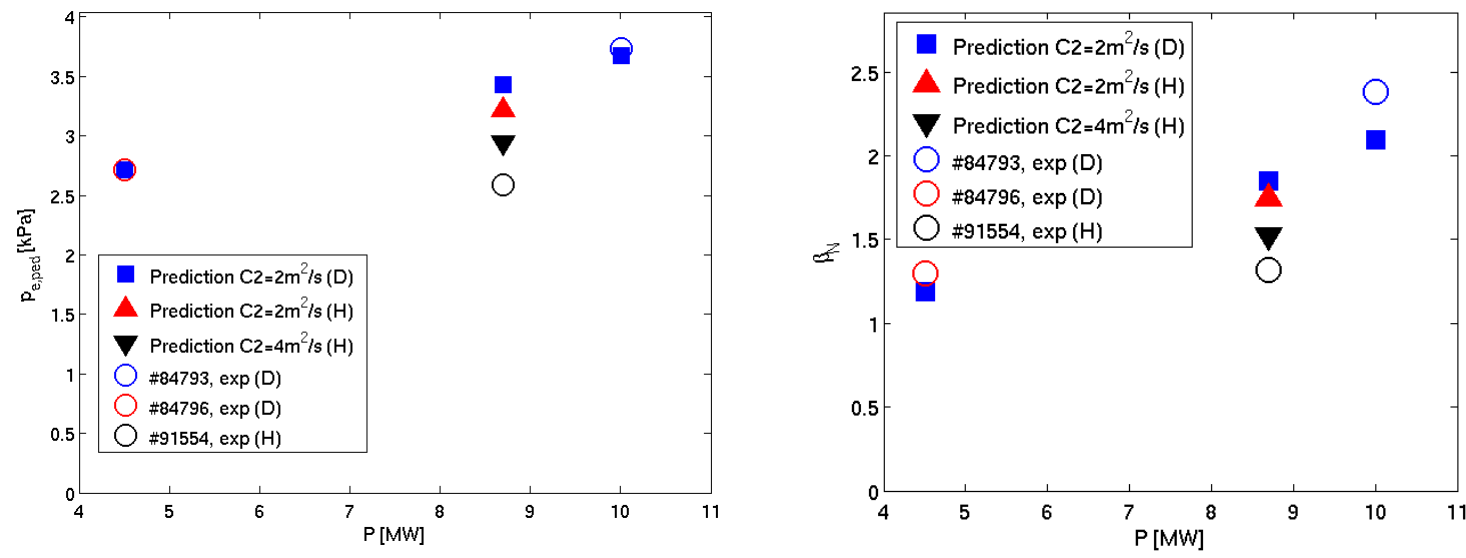

Figure 11 The predicted pedestal pressure (left) and $\beta_{\mathrm{N}}$ (right) for the two deuterium cases (\#84793, low power, and \#84796, high power) and a hydrogen case (\#91554, high power). The different solid symbols represent the different settings in the simulation. ( $\mathrm{H}=$ hydrogen, $\mathrm{D}=$ deuterium). $\mathrm{C} 2$ refers to the coefficient in the critical gradient transport model, Eq. 10. 


\section{Predictions for the DT operation}

The fusion power in the DT operation of JET is highly sensitive to the ion temperature profile in the core. The core transport model used in the pedestal prediction assumes $T_{i}=T_{e}$, which while adequate for the purposes of predicting the pedestal, may lead to a strong underprediction of the core ion temperature, and, consequently the fusion power in the case that ion temperature exceeds electron temperature as was the case in the hot ion mode plasmas of the JET DTE1 campaign where the core ion temperature was more than double of that of the electrons leading to the record fusion power of 16 MW [37]. However, since the heating by $\alpha$-particles plays only a small role, we can still make predictions of the pedestal with the models presented in this paper. As a starting point we use a JETILW discharge \#86614, which was done with $\mathrm{I}_{\mathrm{p}}=2.5 \mathrm{MA}, \mathrm{B}_{\mathrm{t}}=2.9 \mathrm{~T}$ and $\mathrm{P}_{\mathrm{tot}}=25 \mathrm{MW}$. This discharge has low triangularity $(\delta=0.21)$. For comparison we make the prediction also for a high triangularity $(\delta=0.36)$ shape. We use the critical temperature gradient model for core heat transport (Eqs. 9-10 with $(R \nabla T / T)_{e, \text { crit }}=5.0, \mathrm{C} 2=1.6 \mathrm{~m}^{2} / \mathrm{s}$ assuming linear dependency of $\mathrm{C} 2$ with isotope mass $)$, density peaking model (Eq. 11) and modified neutral penetration model (Eqs. 2-7 with $E=2.4 \delta^{-0.53} \phi^{-0.20}$ ) for the pedestal density prediction. To simulate the uncertainty of core ion transport, we do two sets of predictions with $(R \nabla T / T)_{i, c r i t}=(R \nabla T / T)_{e, c r i t}$ and $(R \nabla T / T)_{i, c r i t}=1.5(R \nabla T / T)_{e, c r i t}$ We conduct a scan of the heating power up to the expected maximum available in the DT experiments, $40 \mathrm{MW}$.

The result of the power scan is shown in Fig 12. As expected, the total plasma $\beta_{\mathrm{N}}$ increases with heating power. It is also increased by increasing the triangularity and improved core ion confinement. On the other hand, the pedestal prediction is relatively robust for the ion transport model with only a minor increase in $p_{e, p e d}$ with the improved ion transport. Increasing the heating power increases $p_{e, p e d}$ modestly through the stability improvement by increasing Shafranov shift. Increasing the plasma triangularity increases the predicted $\mathrm{p}_{\text {e.ped }}$ by about $50 \%$ and the predicted $\mathrm{n}_{\mathrm{e} \text {,ped }}$ about $30 \%$, which is the largest effect. The experimental case at $\delta=0.21, \mathrm{P}_{\text {tot }}=25 \mathrm{MW}$ is relatively well predicted for all the parameters. The predicted thermal fusion power varies between $2 \mathrm{MW}$ and $7 \mathrm{MW}$ at $\mathrm{P}_{\text {tot }}=40 \mathrm{MW}$. The variation is almost entirely dominated by the ion transport model with the shape having only a minor effect. This indicates that while the model presented here can give a relatively robust predictions for the pedestal parameters in JET DT plasmas, it is insufficient for predicting the fusion power accurately.
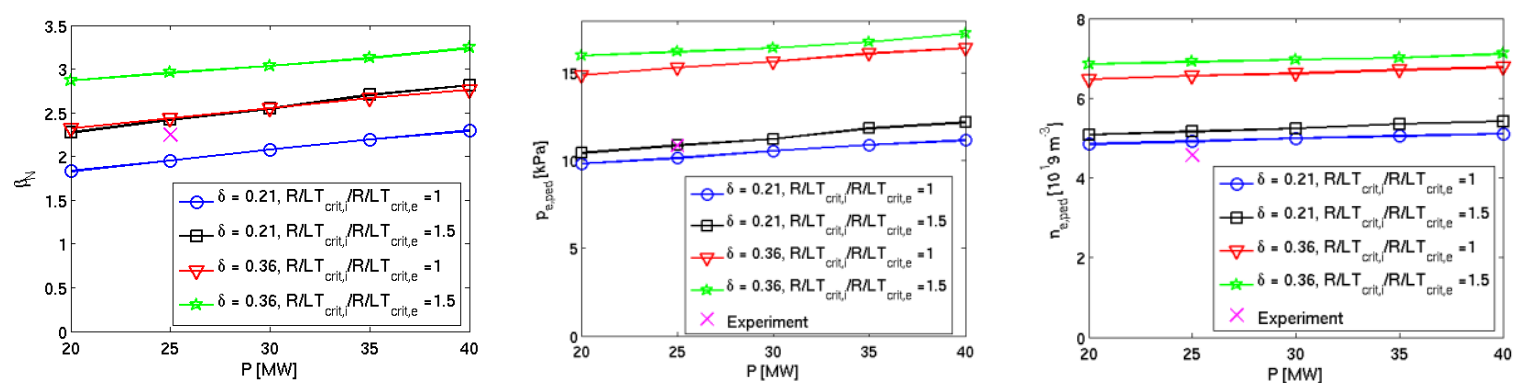

Figure 12. Prediction of $\beta_{\mathrm{N}}$ (left) $\mathrm{p}_{\mathrm{e} \text {,ped }}$ (middle) and $\mathrm{n}_{\mathrm{e} \text {,ped }}$ (right) in the JET-ILW DT experiment as a function of heating power. The predictions are done for four cases: $\delta=0.21, \mathrm{~T}_{\mathrm{e}}=\mathrm{T}_{\mathrm{i}}$ (blue circles), $\delta=0.36, \mathrm{~T}_{\mathrm{e}}=\mathrm{T}_{\mathrm{i}}$ (red triangles), $\delta=0.21,(R \nabla T / T)_{i, c r i t}=1.5(R \nabla \mathrm{T} / T)_{e, c r i t}$ (black squares) and $\delta=0.36$, $(R \nabla T / T)_{i, c r i t}=1.5(R \nabla T / T)_{e, c r i t}$ (green stars).

\section{Summary and Conclusions}


Only about $40 \%$ of the JET-ILW pedestal database plasmas were found to be limited by the PBMs in ideal MHD stability analysis. Self-consistent core-pedestal predictions with a very simple core transport model with a critical gradient were able to match the experimental pedestal pressure for the JET-ILW database cases that were near the PBM stability limit with RMSE $=17 \%$, which is only slightly higher than what is obtained in predictions with a known global $\beta_{\mathrm{N}}(\mathrm{RMSE}=13 \%)$. The match of the electron core temperature to the experiment was improved from that of the predictions done using $\beta_{\mathrm{N}}$ as input.

The neutral penetration model without explicit dependency of the plasma current, toroidal field or the size of the device is able to reach the same level of accuracy in predicting the pedestal top density of deuterium plasmas as what is achieved by parameterising the density with current, field, power, triangularity and fuelling if the corrections of fuelling and especially plasma triangularity are included into the model. The isotope effect inherent in the neutral penetration model predicts that the pedestal density should increase when changing the plasma ions from deuterium to hydrogen, but the opposite is observed in the experiment suggesting that the isotope must have an effect on pedestal particle transport either between ELMs or due to ELMs that more than compensates the effect of deeper penetration of neutrals in hydrogen or that the relatively simple model used is not able to capture all the aspects of the neutral penetration physics.

The combined core-pedestal model and the neutral penetration model without knowledge of the global plasma $\beta$ and density is able to predict the pedestal top pressure almost at the same level of accuracy as the standard EPED1 model (RMSE $=16 \%$ vs RMSE=13\%). One key element still missing from the pedestal model is the relative shift of the density with regards to the temperature profile, which is not predicted. Frassinetti et al. showed that it can influence the pedestal stability in JET-ILW [37]. Developing a physics model for the shift will be left for future work.

The decreased pedestal pressure in hydrogen experiment compared to the deuterium at the similar heating power is reproduced with the self-consistent model when the increase in core heat diffusivity and the isotope effect on the pedestal stability are taken into account in the predictive model. However, the experimental isotope effect on the pedestal density is opposite to what the neutral penetration model predicts and, consequently, the density pedestal cannot be predicted in hydrogen plasmas with it.

For predicting pedestal pressure in the future JET DT plasmas, the simple core transport model is most likely sufficient. However, as the produced fusion power is sensitive to the details of the ion temperature profile, this method is not suitable for its predictions and for that purpose the pedestal prediction should be combined with a more detailed core transport simulation, sophisticated fast ion slowing down models and beam-plasma fusion reaction models, such as those done with JETTO and CRONOS in [39] and [40].

\section{Acknowledgements}

This work has been carried out within the framework of the EUROfusion Consortium and has received funding from the Euratom research and training programme 2014-2018 and 2019-2020 under grant agreement No 633053 and from the RCUK Energy Programme [grant number EP/P012450/1]. The views and opinions expressed herein do not necessarily reflect those of the European Commission. To obtain further information on the data and models underlying this paper please contact PublicationsManager@ukaea.uk

\section{References}


[1] R.J. Groebner, M. A. Mahdavi, A. W. Leonard, T. H. Osborne, G. D. Porter, R. J. Colchin, and L. W. Owen, Phys. Plasmas 9 (2002) 2134

[2] J.G. Cordey et al Nucl. Fusion 43 (2003) 670

[3] L. Frassinetti, S. Saarelma, F. Imbeaux, G. Verdoolaege, P. Bilkova, P. Bohm, R. Fridström, E. Giovannozzi, M. Owsiak, M. Dunne, B. Labit, R. Scannell, J.C. Hillesheim, Proc. of the 45th EPS conference on Plasma Physics, July 2-6 2018, Prague, Czech Republic, P4.1027

[4] P.B. Snyder R. J. Groebner, A. W. Leonard, T. H. Osborne, and H. R. Wilson, Phys. Plasmas, 16, (2009) 056118

[5] P.B. Snyder, R.J. Groebner, J.W. Hughes, T.H. Osborne, M. Beurskens, A.W. Leonard, H.R. Wilson and X.Q. Xu, Nucl. Fusion 51 (2011) 103016

[6] H. Urano, T. Takizuka, Y. Kamada, N. Oyama, H. Takenaga, Nucl. Fusion 48 (2008) 045008

[7] H. Urano, T. Takizuka, M. Kikuchi, T. Nakano, N. Hayashi, N. Oyama, and Y. Kamada, Phys. Rev. Lett. 109, (2012) 125001

[8] F. M. Laggner, E. Wolfrum, M. Cavedon, F. Mink, M. Bernert, M. G. Dunne, P. A. Schneider, A. Kappatou, G. Birkenmeier, R. Fischer, M. Willensdorfer, F. Aumayr, EUROfusion MST1 Team, and ASDEX Upgrade Team, : Phys. Plasmas 24, (2017) 056105

[9] R.J. Groebner, A.W. Leonard, P.B. Snyder, T.H. Osborne, C.F. Maggi, M.E. Fenstermacher, C.C. Petty and L.W. Owen, Nucl. Fusion 49 (2009) 085037

[10] J.G. Cordey, B. Balet, D.V. Bartlett, R.V. Budnya, J.P. Christiansen, G.D. Conway, L.-G. Eriksson, G.M. Fishpool, C.W. Gowers, J.C.M. de Haasb, Nucl. Fusion 39 (1999) 301

[11] C F Maggi, H Weisen, J C Hillesheim, A Chankin, E Delabie, L Horvath, F Auriemma, I S Carvalho, G Corrigan, J Flanagan, L Garzotti , D Keeling, D King, E Lerche, R Lorenzini, M Maslov, S Menmuir, S Saarelma, A C C Sips, E R Solano, E Belonohy, F J Casson, C Challis, C Giroud, V Parail, C Silva , M Valisa, Plasma Phys. Control. Fusion 60 (2018) 014045

[12] R. Pasqualotto, P. Nielsen, C. Gowers, M. Beurskens, M. Kempenaars, Rev. Sci. Instrum. 75, (2004) 3891

[13] L. Frassinetti, M.N.A. Beurskens, R. Scannell, T.H. Osborne, J. Flanagan, M. Kempenaars, M. Maslov, R. Pasqualotto, M. Walsh, Rev. Sci. Instrum, 83 (2012) 01350613

[14] I Nunes and the JET Contributors, Plasma Phys. Control. Fusion, 58 (2016) 014034

[15] A. Kallenbach, N. Asakura, A. Kirk, A. Korotkov, M.A. Mahdavi, D. Mossessian, G.D. Porter, Jour. of Nucl, Mat. 337-339 (2005) 381

[16] S Saarelma, A Alfier, M N A Beurskens, R Coelho, H R Koslowski, Y Liang, I Nunes and JET EFDA contributors, Plasma Phys. Control. Fusion 51 (2009) 035001

[17] G T A Huysmans, J P Goedbloed and W O K Kerner 1991 Computational Physics Proc. Int. Conf. (Amsterdam, 1991) (Singapore: World Scientific Publishing) p 371 
[18] P.B. Snyder, H. R. Wilson, J. R. Ferron, L. L. Lao, A. W. Leonard, T. H. Osborne, A. D. Turnbull, D. Mossessian, M. Murakami, and X. Q. Xu Phys. Plasmas 9 (2002) 2037

[18] H.R. Wilson, P.B. Snyder, G.T.A. Huysmans and R.L. Miller, Phys. Plasmas 9 (2002) 1277

[20] N. Aiba, C. Giroud, M. Honda, E. Delabie, S. Saarelma, L. Frassinetti, I. Lupelli, F.J. Casson, S. Pamela, H. Urano, C.F. Maggi and JET Contributors, Nucl. Fusion 57 (2011) 126001

[21] M.J. Leyland, M.N.A. Beurskens, L. Frassinetti, C. Giroud, S. Saarelma, P.B. Snyder, J. Flanagan, S. Jachmich, M. Kempenaars, P. Lomas, G. Maddison, R. Neu, I. Nunes, K.J. Gibson, Nucl. Fusion 55 (2015) 013019

[22] H. Urano, J. Hobirk, C.F. Maggi, E. Joffrin, Proc. 43rd EPS Conference on Plasma Physics, 4 - 8 July, 2016, Leuven, Belgium, O4.121

[23] A. Kirk, G.F. Counsell, E. Arends, H. Meyer, D. Taylor, M. Valovic, M. Walsh, H. Wilson and the MAST team Plasma Phys. Control. Fusion 46 (2004) A187

[24] R.L. Freeman and E.M. Jones. Atomic collision processes in plasma physics experiments. (CLMR 137), May 1974

[25] P. B. Snyder, K. H. Burrell, H. R. Wilson, M. S. Chu, M. E. Fenstermacher, A. W. Leonard, R. A. Moyer, T. H. Osborne, M. Umansky, W. P. West, and X. Q. Xu, Nucl. Fusion 47 (2007) 961

[26] S.K. Kim Y.-S. Na, S. Saarelma and O. Kwon, Nucl. Fusion 58 (2018) 016036

[27] G. Staebler, J. Kinsey, and R. Waltz, Phys. Plasmas 14, (2007) 055909.

[28] J.E. Kinsey, G.M. Staebler, J. Candy, R.E. Waltz and R.V. Budny, Nucl. Fusion 51 (2011) 083001

[29] O. Meneghini, P. B. Snyder, S. P. Smith, J. Candy, G. M. Staebler, E. A. Belli, L. L. Lao, J. M. Park, D. L. Green, W. Elwasif, B. A. Grierson, and C. Holland, Physics of Plasmas 23, (2016) 042507

[30] P. Mantica, D. Strintzi, T. Tala, C. Giroud, T. Johnson, H. Leggate, E. Lerche, T. Loarer, A. G. Peeters, A. Salmi, S. Sharapov, D. Van Eester, P. C. de Vries, L. Zabeo, and K.-D. Zastrow, Phys. Rev. Lett. 102 (2009) 175002

[31] A. M. Dimits, G. Bateman, M. A. Beer, B. I. Cohen, W. Dorland, G. W. Hammett, C. Kim, J. E. Kinsey, M. Kotschenreuther, A. H. Kritz, L. L. Lao, J. Mandrekas, W. M. Nevins, S. E. Parker, A. J. Red, D. E. Shumaker, R. Sydora, J. Weiland, Phys. Plasmas 7 (2000) 969

[32] F. Jenko, W. Dorland, and G. W. Hammett, Phys Plasmas, 8 (2001) 4096

[33] C. Angioni, H. Weisen, O.J.W.F. Kardaun, M. Maslov, A. Zabolotsky, C. Fuchs, L. Garzotti, C. Giroud, B. Kurzan, P. Mantica, A.G. Peeters, J. Stober, Nucl. Fusion 47 (2007) 1326

[34] V.P. Bhatnagar, J. Lingertat, R. Barnsley, P. Breger, J.P. Christiansen, S. Clement, J.G. Cordey, S.J. Davies, J.K. Ehrenberg, L.-G. Eriksson, G.M. Fishpool, P.J. Harbour, L.D. Horton, J. Jacquinot, H.J. Jäckel, K. Lawson, C.G. Lowry, C.F. Maggi, G.F. Matthews, R.D. Monk, D.P. O'Briena, V.V. Parail, E. Righid, G. Saibene, R. Sartori, B. Schunke, A.C.C. Sips, M.F. Stamp, D.F.H. Start and K. Thomsen Nucl. Fusion, 39 (1999) 353 
[35] G. Saibene, L.D. Horton, R. Sartori, B. Balet, S. Clement, G.D. Conway, J.G. Cordey, H.P.L. De Esch, L.C. Ingesson, J. Lingertat, R.D. Monk, V.V. Parail, R.J. Smith, A. Taroni, K. Thomsen, M.G. von Hellermann, Nucl. Fusion 39 (1999) 1133

[36] L. Horvath et al. to be submitted to Nucl. Fusion

[37] L.Frassinetti , M.G. Dunne, U. Sheikh, S. Saarelma, C. M. Roach, E.Stefanikova, C. Maggi , L. Horvath, S. Pamela, E. de la Luna, E. Wolfrum, M. Bernert, P. Blanchard, B. Labit, A. Merle, L. Guimarais, S. Coda, H. Meyer, J.C. Hillesheim et al. accepted for publication in Nucl. Fusion

[38] M. Keilhacker, A. Gibson, C. Gormezano, P.J. Lomas, P.R. Thomas, M.L. Watkins, P. Andrew, B. Balet, D. Borba, C.D. Challis, et al., Nucl. Fusion 39 (1999) 209

[39] J Garcia, C Challis, D Gallart, L Garzotti, T Görler, D King, M Mantsinen, Plasma Phys. Control. Fusion 59 (2017) 014023

[40] J. Garcia, R.J. Dumont, J. Joly, J. Morales, L. Garzotti, T,W, Bache, Y. Baranov, F.J. Casson, C. Challis, K. Kirov, J. Mailloux, S. Saarelma et al. accepted for publication in Nucl. Fusion 